\title{
Dynamics of surface magnetization on a nanosecond time scale
}

\author{
Fausto Sirotti \\ Laboratoire pour l'Utilisation du Rayonnement Electromagnetique, CNRS-CEA-MESR, F-91405 Orsay, France \\ Simone Girlando \\ INFM, Dipartimento di Fisica dell'Università di Modena, I-4100 Modena, Italy \\ Pilar Prieto \\ Departamento Fisica Aplicada, C-XII, Facultad de Ciencias, Universidad Autonoma de Madrid, E-28049 Cantoblanco, Spain \\ Luca Floreano \\ INFM, TASC Laboratory, Area di Ricerca, Padriciano 99, Trieste, Italy \\ Giancarlo Panaccione \\ APE Project, INFM, TASC Laboratory, Area di Ricerca, Padriciano 99, Trieste, Italy \\ Giorgio Rossi \\ INFM, Dipartimento di Fisica dell'Università di Modena, I-4100 Modena, Italy \\ and Laboratorium für Festkörperphysik, ETH-Zürich, CH-8093 Zürich, Switzerland
}

(Received 5 November 1999)

\begin{abstract}
The dynamics of surface magnetization is measured with ns time resolution by spin-polarimetry of the total photoemission yield excited by synchrotron radiation pulses. The surface response is compared to the bulk magnetization dynamics as obtained by induction measurements. The surface and the bulk show distinct magnetization dynamics indicating weak coupling during the reversal process in the ns- $\mu$ s time domain. Ultrathin layers of $\mathrm{Fe}$ as well as three-layer $\mathrm{Fe} / \mathrm{Cu} / \mathrm{Fe}$ exchange coupled structures were grown on top of an amorphous soft-ferromagnetic substrate (Vitrovac) and showed different reversal dynamics.
\end{abstract}

The surface of a ferromagnet has a special magnetic behavior with respect to the bulk. ${ }^{1,2}$ The electronic structure of the surface layer implies that exchange is anisotropic, i.e., the exchange stiffness is different on a path within the layer compared to a path perpendicular to it. ${ }^{3}$ The surface magnetization of the $3 d$ ferromagnetic metals is characterized by enhanced spin and orbital magnetic moments with respect to the bulk. ${ }^{4,5}$ The critical behavior of the magnetization near the Curie temperature $\left(T_{C}\right)$ is described by surface critical exponents which differ from the bulk ones. The ferromagnetic/paramagnetic transition itself may occur at a different temperature with respect to the bulk $T_{C} \cdot{ }^{6}$ Based on these considerations alone one expects the dynamics of magnetization and of magnetization reversal at a ferromagnetic surface to be different than in the bulk.

Our experiments are based on the surface sensitivity of the measurement of spin polarization (SP) of the photoemission yield as excited by synchrotron radiation (SR) in the UV and soft x-ray energy range, and on the pulsed structure of the radiation from an undulator source on a positron storage ring. 500 ps-long pulses of SR at time intervals of $120 \mathrm{~ns}$, were obtained exploiting the "two bunch mode" of the SuperAco storage ring at Orsay. The polychromatic undulator radiation or monochromatic radiation of energy $h \nu=200 \mathrm{eV}$ from the undulator source DOMINO at SuperAco was focused to a spot of about $3 \times 1 \mathrm{~mm}$ and impinged on the sample surface. This sets the lateral scale of the experiment which is therefore representative of a macroscopic magnetic behavior of the surface, i.e., integrated over the domain structure. Atomically clean surfaces of a soft-magnetic ribbon, as well as iron monolayers or iron/copper/iron interfaces, were prepared by ion sputtering and $e$-beam evaporation techniques in an ultrahigh vacuum environment, and measured at room temperature. The primary and secondary photoejected electrons from the sample surface were collected by an electrostatic accelerator lens and directed to the thin Au target of a $100 \mathrm{KV}$ Mott scattering detector. The spin polarization of the ejected electron beam $\mathrm{SP}=\left(I_{u p}\right.$ $\left.-I_{\text {down }}\right) /\left(I_{\text {up }}+I_{\text {down }}\right)$, where $I_{\text {up (down) }}$ are the spin-up or -down intensities, was measured and independently registered after each pulse of synchrotron radiation, i.e., at 8.333 $\mathrm{MHz}$ rate. ${ }^{7,8}$ The measure of the bulk magnetization reversal dynamics was obtained by means of an induction search coil in otherwise identical experimental and timing conditions.

The external magnetic field was applied to the whole sample by means of a current pulse in a low inductance coil. A steady magnetization state (saturation) was maintained by a constant bias current in the coil. At the time $t=0$ of each experiment the current in the coil was reversed and set to a fixed value of choice (between a few $\mathrm{mA}$ up to about 40 amperes). The reversed applied field stabilized typically within $\sim 70 \mathrm{~ns}$, after which it remained constant during the data acquisition. In Fig. 1 we present a scheme of the time structure of the experiment with the SR pulses at $120 \mathrm{~ns}$ intervals (real time mode). By applying a delay of $1 \mathrm{~ns}$ or multiples to the magnetizing pulse with respect to the SR 


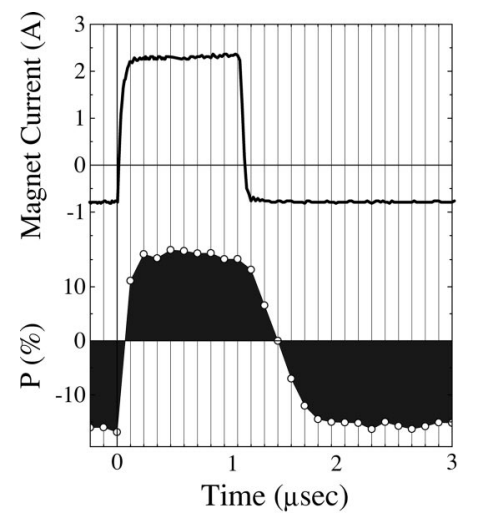

FIG. 1. Time structure of the experiments: The grid represents the SR pulses (500 ps every $120 \mathrm{ns).} \mathrm{The} \mathrm{upper} \mathrm{curve} \mathrm{is} \mathrm{the} \mathrm{current}$ in the magnetic circuit, triggered on a SR pulse at $t_{0}$. The lower data are SP measurements for zero delay with respect to $t_{0}$. Delays of 1 ns or longer have been applied shifting the data acquisition with respect to the applied field.

pulses and by repeating the experiment the overall time resolution of about $1 \mathrm{~ns}$ is obtained (pump and probe mode). ${ }^{7,8}$

In Fig. 2 we present the surface sensitive and bulk sensitive magnetization data as a function of time $t$ after the application of an external field antiparallel to the previous saturated magnetization state $\left(t_{0}\right)$. Various field magnitudes were applied to the sample: they are given in units of the coercive field $H_{c 0}$ as measured in a standard (i.e., slow) hysteresis loop for the Vitrovac substrate. All curves have the same general shape: the demagnetization state (zero spin polarization in the surface data) is reached at a time $t_{D}$ and the reversed saturation magnetization is reached in a time exceeding $2 t_{D}$. The analysis of these $M(t)$ curves could be attempted to some extents by means of models involving energy barriers ${ }^{9}$ physically related to domain nucleation and domain-wall motion. ${ }^{10,11}$ This kind of analysis leads to a large set of fitting parameters whose physical meaning is vague and to the conclusion that the local domain-wall motion is governed by viscous motion in an external magnetic

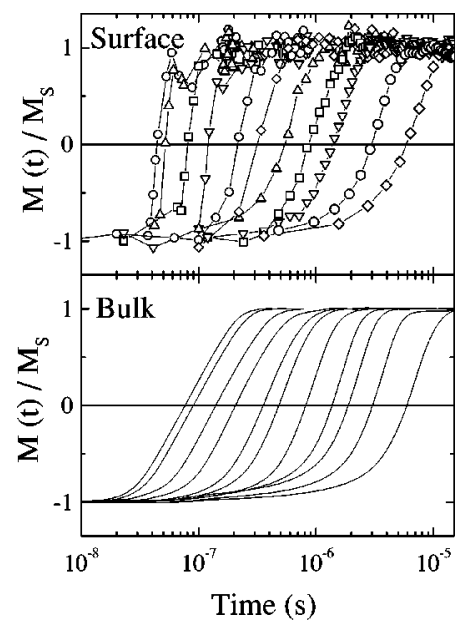

FIG. 2. Magnetization reversal curves measured on a $100 \mu \mathrm{m}$-thick Vitrovac sample for the surface (upper panel) and the bulk (lower panel). The curves are obtained applying a field 8 , $12,20,28,50,84,112,209,331,477$, and 654 times larger than $H_{c 0}$.

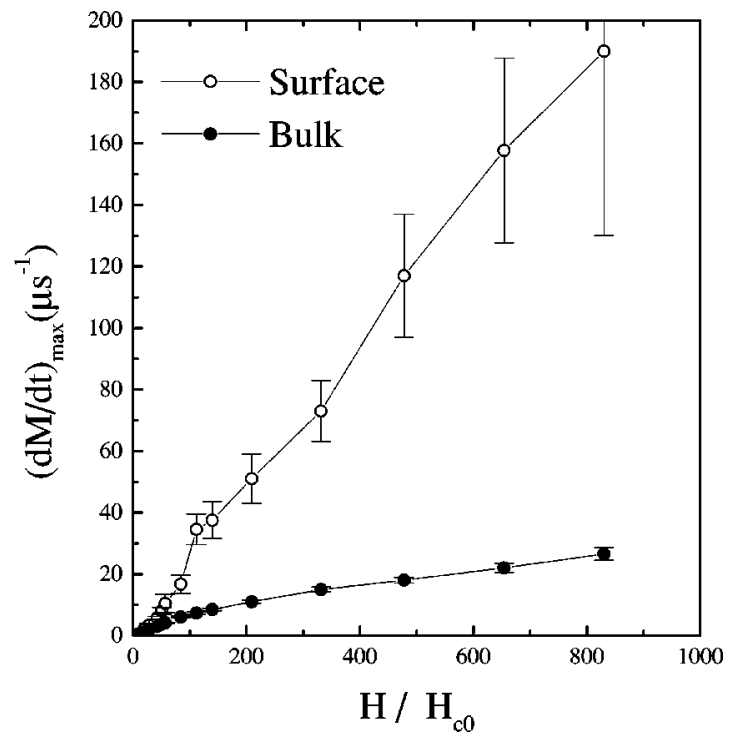

FIG. 3. Maximum slope of the magnetization reversal curves as a function of the applied magnetic field obtained for the surface (open symbols) and the bulk (solid symbols).

field. ${ }^{14}$ By comparing the surface and bulk experimental curves for the same applied field it is found that the surface magnetization reversal of a $100 \mu \mathrm{m}$-thick Vitrovac ribbon advances the bulk one: the inequality $t_{D S u r f}<t_{D B u l k}$ becomes progressively larger as the applied field increases and the reversal time decreases. The magnetization reversal speed can also be represented by the value of the time derivative of the magnetization reversal curves evaluated at $t_{D}$ as shown in Fig. 3. The maximum magnetization reversal speed as well as the dynamical coercivity of bulk and surface appear quite different in the high magnetic-field regime. An applied field of 130 times $H_{c 0}$ is sufficient to switch the magnetization of the surface with $t_{D}=110 \mathrm{~ns}$ and a maximum speed of $37 \mu \mathrm{s}^{-1}$, but the bulk magnetization reversal process takes place with $t_{D}=180 \mathrm{~ns}$ and maximum speed of $8 \mu \mathrm{s}^{-1}$. In the time interval $t_{D S u r f}<t<t_{D B u l k}$ the net orientation of surface and bulk are therefore opposite. This important point must be addressed carefully since the bulk reversal data are affected by the induction of eddy currents as the reversal proceeds and the effect of the demagnetizing field for a steplike reversal of the applied field is hard to evaluate quantitatively. On the other hand, the comparison of the SP data measured for different surfaces grown on the same Vitrovac substrate, and therefore exchange coupled to it, is independent on the actual applied field and magnetization dynamics of the substrate.

The thermal decrease of the surface magnetization is larger compared to the bulk due to the double probability of finding spin waves at the surface than in the bulk. ${ }^{3}$ Various experiments have been performed to establish the spin-wave stiffness of surfaces, including permalloy and $\mathrm{Fe}(100) .{ }^{15-17} \mathrm{It}$ has been found that indeed the exchange interaction along a path perpendicular to the surface is reduced and that it can be further modified by modifying the chemical composition or the structure of the surface. This means that the surface is "weakly" exchange coupled to the bulk and that this coupling can be artificially modified. A further hint to the weak coupling of surface and bulk was given by the comparison of 


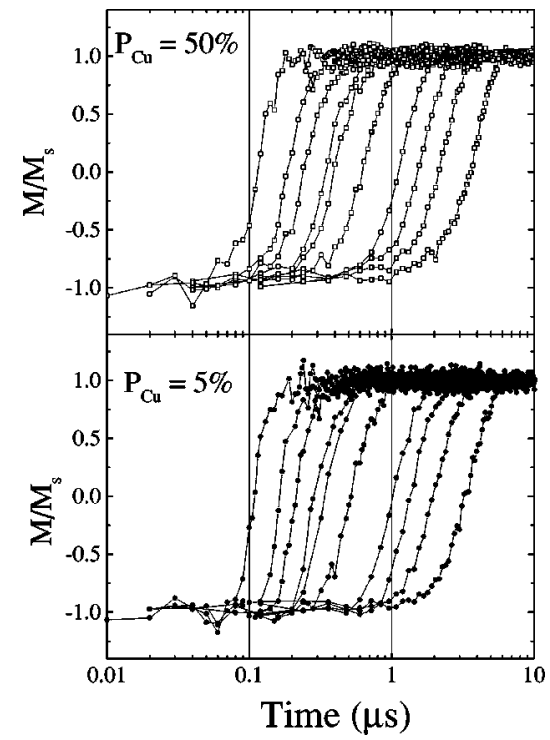

FIG. 4. Surface magnetization reversal curves measured on the surface of a three-layer $\mathrm{Fe} / \mathrm{Cu} / \mathrm{Fe}$ system. The copper thickness was $4 \AA$ (upper panel) and $10 \AA$ (lower panel). The curves are obtained applying a field $12,16,20,24,32,40,48,60,80$, and 160 times larger than $H_{c 0}$.

surface and bulk hysteresis loops. ${ }^{12}$ We have investigated the magnetization dynamics of exchange coupled structures by comparing $\operatorname{SP}(t)$ data for different surfaces deposited onto atomically clean Vitrovac: nanometer-thick layers of iron and $\mathrm{Fe} / \mathrm{Cu} / \mathrm{Fe}$ three-layer structures with different layer thicknesses. The bottom and top iron layers were $20 \AA$ thick and the copper spacers were $4 \AA$ and $10 \AA$ thick. The SP of secondary electrons measured after the deposition of the copper spacer layer was $50 \%$ and $5 \%$ of the value measured for the bottom iron layer, respectively . The magnetization reversal curves are shown in Fig. 4 where the results obtained for the $4 \AA$ and $10 \AA$ Cu thicknesses are identified by the SP values across the spacer. Equilibrium experiments show that the Fe surface is exchange coupled parallel to the Vitrovac surface and that it displays a square hysteresis loop with the same coercive field as the substrate. The upper iron layer is exchange coupled to the substrate through the copper intralayer, but the coupling vanished for $\mathrm{Cu}$ thicknesses larger than $20 \AA$.

The comparison between the maximum magnetization reversal speed of the two Fe surfaces is shown in Fig. 5. The magnetization reversal process of the less coupled Fe layer is faster then the more strongly coupled one. The experimental curves measured for an applied field 80 times larger than $H_{c 0}$ are shown in Fig. 6. It appears that the ferromagnetic surfaces follow different time patterns showing also a variable delay of the onset of reversal. In particular, the less coupled $\mathrm{Fe} / \mathrm{Cu} / \mathrm{Fe}$ surface layer (open symbols) presents a magnetization reversal transition with a delayed onset but a faster transition which gives a reduced $t_{D S u r f}$ value.

The demonstration and understanding of exchange coupled artificial structures is at the basis of current magnetic multilayer technology. An iron film can be made magnetically "soft" by exchange coupling to a soft-ferromagnet substrate such as Permalloy or Vitrovac. One obtains in that case the high magnetic moment of pure iron and the very

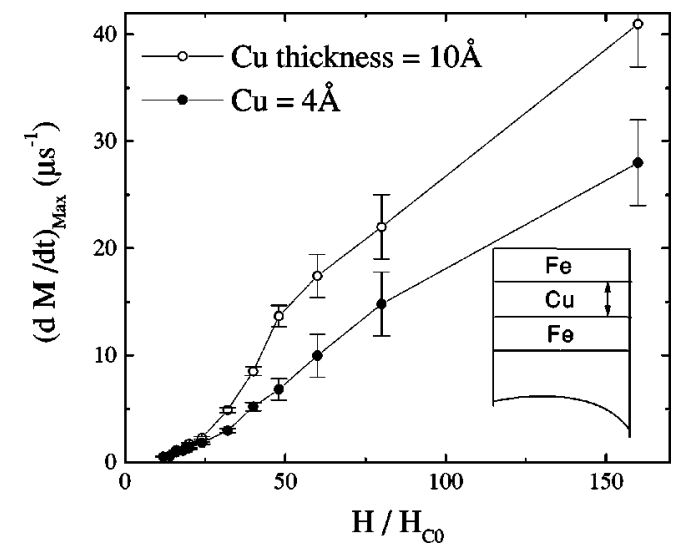

FIG. 5. Maximum slope of the surface magnetization reversal curves measured on the surface of two exchange coupled system: $(20 \AA \mathrm{Fe}) /(4 \AA \mathrm{Cu}) /(20 \AA \mathrm{Fe})$ and $(20 \AA \mathrm{Fe}) /(10 \AA \mathrm{Cu}) /(20 \AA \mathrm{Fe})$ vs the applied field.

narrow hysteresis of Permalloy or Vitrovac which is therefore called the magnetic "driver" of the surface iron film. The present data show that, by inserting an intralayer of a nonmagnetic material such as copper, one can control the reduction of the magnetic coupling between the top iron layer and the substrate and this is reflected in a very different relative dynamical behavior. The $\mathrm{Fe} / \mathrm{Cu} / \mathrm{Fe}$ system shows a delay of the onset but a faster reversal transition with respect to the more strongly coupled Fe layer and Vitrovac surfaces.

The results of an independent experiment addressing the surface to bulk dynamical coupling are shown in Fig. 7. Here the dynamical response of the magnetization of a Vitrovac film to an applied field of $12 H_{c 0}$ is studied as a function of the time duration $t_{n e g}$ of the previous opposite magnetizing field whose amplitude is -32 times $H_{c 0}$. The time scale of the magnetization reversal processes is aligned taking $t=0$ when the $-32 H_{c 0} /+12 H_{c 0}$ field inversion takes place. The SP data show that the reversal dynamics is extremely dependent on $t_{\text {neg }}$, that is on the previous history of the sample. For a very short $t_{n e g}=0.48 \mu \mathrm{s}$ the surface had not reached the saturation before the new reversal was induced.

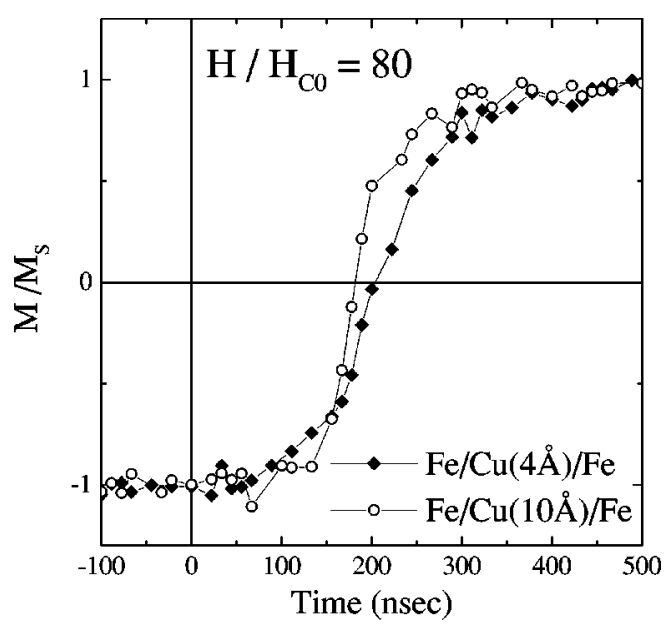

FIG. 6. Magnetization reversal curves measured for an applied field $80 H_{c 0}$ for two $\mathrm{Fe} / \mathrm{Cu} / \mathrm{Fe}$ systems for which the SP after $\mathrm{Cu}$ deposition was reduced to $50 \%$ and $5 \%$ of the SP of the clean iron substrate. 


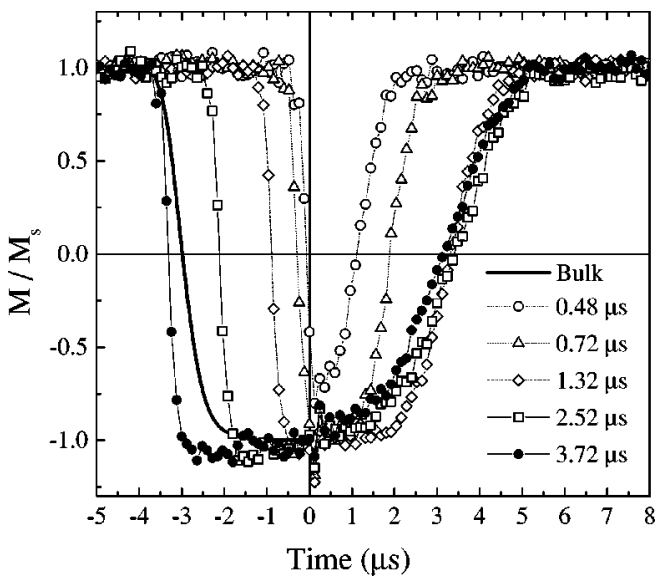

FIG. 7. Surface magnetization reversal curves obtained for negative pulse duration ranging between $0.48 \mu \mathrm{s}$ and $3.72 \mu \mathrm{s}$. The magnetic field values are $-32 H_{c 0}$ before $t_{0}$ and $12 H_{c 0}$ after $t_{0}$. The continuous line represents bulk magnetization reversal curve for the transition to $-32 \mathrm{H}_{c 0}$.

But for values of $t_{n e g} \geqslant 0.72 \mu \mathrm{s}$ the surface was fully saturated. The reversal dynamics nevertheless is very different for values of pulsewidth up to $3.72 \mu \mathrm{s}$ (solid symbols in Fig. 7). For $1.3 \mu \mathrm{s} \leqslant t_{n e g} \leqslant 3.7 \mu \mathrm{s}$ the magnetization reversal process has a delayed onset but a higher speed with respect to the behavior measured in equilibrium conditions. The corresponding bulk dynamics is shown by the thick solid line. These results are quite important since they show that the bulk cannot reach an equilibrium state, with the present value of applied field, before $3.72 \mu \mathrm{s}$. This phenomenon could be explained by a domain-structure-shape memory effect as in Ref. 14. The "weak" surface to bulk coupling is clearly observed in this experiment: the surface magnetization reversal is the slowest when at $t=0$ both bulk and surface are saturated, but it is much faster when at $t=0$ the bulk had not yet reached saturation in the previous magnetization direction. After the shortest pulse that produces surface satu- ration, in a out-of-equilibrium state of the ferromagnet, the next surface reversal is the fastest, with a doubled speed with respect to the case of initial surface-bulk saturation equilibrium state. This behavior mimics a spring coupling between the surface and bulk magnetization. This experiment independently confirms that the magnetization dynamics at the surface is faster than in the bulk and removes all uncertainties connected to the effective value of the applied field inside the bulk. It does show that, during reversal, the surface and bulk of a ferromagnet are two subsystems out of equilibrium.

The "weak coupling" between the surface and the bulk in ferromagnets is put in evidence by the present experiments on the dynamics of the surface magnetization reversal in the $100-1000 \mathrm{~ns}$ time scale. The details of the magnetization reversal mechanism are not directly retrievable from the experiments. The fact that the magnetization reversal starts promptly at the surface is possibly related to the noncollinear alignment of the surface and bulk magnetic moment due to the surface anisotropy as suggested in Refs. 12 and 13. As the reversed field is applied, the torque exerted on the magnetic moments can be finite only for surface moments, if they are even slightly misaligned with respect to the bulk moments. The bulk magnetization reacts then to the surface reversal with a spring-coupling behavior. Structural and domain distribution informations are needed in order to attempt a useful micromagnetics analysis of this phenomenon, but the present results clearly show how surface magnetization reversal is different with respect to bulk magnetization reversal.

The main message of the present experiments is that the surface magnetization is not in equilibrium with the bulk magnetization when a sudden change of applied field occurs. Modified surfaces show different dynamical response to external fields but always appear to switch faster than the bulk of a $100 \mu \mathrm{m}$-thick amorphous soft ferromagnet.

We thank Ch. Back for discussion and H. C. Siegmann for stimulating suggestions and continuous support.
${ }^{1}$ H.C. Siegmann, J. Phys.: Condens. Matter 4, 8935 (1992).

${ }^{2}$ U. Gradmann, in Handbook of Magnetic Materials, edited by K.H. Buschow (Elsevier Science Publishers, 1993), Vol. 7.

${ }^{3}$ J. Mathon and S.B. Ahmad, Phys. Rev. B 37, 660 (1988).

${ }^{4}$ C.L. Fu et al., Phys. Rev. Lett. 54, 2700 (1985).

${ }^{5}$ G. Panaccione, F. Sirotti, and G. Rossi, Solid State Commun. (to be published).

${ }^{6}$ F. Gauthier, in Rayonnement Synchrotron Polarisé Electrons Polarisés et Magnetisme, edited by E. Beaurepaire, B. Carrière, and J.P. Kappler (IPCMS, Strasbourg, 1989).

${ }^{7}$ F. Sirotti, R. Bosshard, P. Prieto, G. Panaccione, L. Floreano, A. Jucha, J.D. Bellier, and G. Rossi, J. Appl. Phys. 83, 1563 (1998).

${ }^{8}$ S. Girlando, thesis (Tesi di Laurea), Università di Modena e Reggio Emilia, 1998.

${ }^{9}$ R.H. Victora, Phys. Rev. Lett. 63, 457 (1989).

${ }^{10}$ M. Labrune, S. Andrieu, F. Rio, and P. Bernstein, J. Magn. Magn.
Mater. 80, 211 (1989).

${ }^{11}$ B. Raquet, R. Mamy, and J.C. Ousset, Phys. Rev. B 54, 4128 (1996).

${ }^{12}$ R. Allenspach, M. Taborelli, M. Landolt, and H.C. Siegmann, Phys. Rev. Lett. 56, 953 (1986).

${ }^{13}$ A.P. Popov and D.P. Pappas, Phys. Rev. B 56, 3222 (1997).

${ }^{14}$ J. Ferré, V. Grolier, P. Meyer, S. Lemerle, A. Maziewski, E. Stefanowicz, S.V. Tarasenko, V.V. Tarasenko, M. Kisielewski, and D. Renard, Phys. Rev. B 55, 15092 (1997).

${ }^{15}$ H.C. Siegmann, D. Mauri, D. Scholl, and E. Kay, J. Phys. (Paris), Colloq. 49, C8-9 (1988).

${ }^{16}$ D. Mauri, D. Scholl, H.C. Siegmann, and E. Kay, Phys. Rev. Lett. 61, 758 (1988).

${ }^{17}$ F. Sirotti, G. Panaccione, and G. Rossi, Phys. Rev. B 52, R17 063 (1995). 